\title{
Korpora als Schlüssel zur lexikografischen Überarbeitung - der neue DoRNSEIFF
}

\author{
$1 \quad$ Allgemeines \\ 2 Korpusgrundlage \\ 3 Erweiterungen und Aktualisierungen \\ 3.1 Mikrostrukturelle Veränderungen \\ 3.2 Vollständiges alphabetisches Register \\ 4 Layout
}

\author{
5 CD-ROM-Version \\ 5.1 Daten-Retrieval \\ 5.2 Verlinkung \\ 5.3 Textverarbeitungselemente \\ 6 Bibliographie \\ 6.1 Wörterbücher \\ 6.2 Literatur
}

\begin{abstract}
Seit 1970 sind mehrere Versuche gemacht worden, den DoRNSEIFF in einer neuen Bearbeitung herauszubringen. Aber eine Überarbeitung, selbst wenn sie aus den alten 20 Hauptklassen 15 oder auch 30 machen und sonst noch viel ändern würde, scheint auf unüberwindliche Schwierigkeiten zu stoßen.
\end{abstract}

(HASS-ZuMKeHR 2001: 288)

\section{Allgemeines}

Mit der 8. Auflage des DoRnSEIFF (DoRnSEIFF 2004) liegt erstmals eine völlig neu überarbeitete Ausgabe dieses nach Sachgruppen geordneten Wörterbuches seit 1959 vor. Die Neubearbeitung dauerte vier Jahre und konnte laut QuAsTHOFF (2004) aufgrund maschineller Methoden eine Zeitersparnis von $90 \%$ verzeichnen. ${ }^{1}$ Auf der Basis einer elektronisch gespeicherten Textsammlung, die mit einem Textanalysetool recherchierbar ist, werden Informationen über einen jeweils repräsentierten Wortschatzausschnitt gewonnen, die sich in quantitativer und qualitativer Hinsicht deutlich von der kompetenzgesteuerten Datengewinnung unterscheiden. Der neue DoRNSEIFF lässt vor allem eins deutlich werden: Es ist möglich, ein längst überholtes Sachgruppenwörterbuch systematisch zu überarbeiten, es zu aktualisieren und angemessen zu erweitern, wenn man über eine aktuelle Datenbasis verfügt und sich der neuen lexikografischen Methoden bedient. Nur durch computertechnologische Unterstützung ist es heute möglich, die eingangs genannten unüberwindlichen Schwierigkeiten nach über 30 Jahren zu bewältigen.

Neben der Neuerarbeitung des begriffssystematischen Wörterverzeichnisses wurde der DoRNSEIFF um eine interessante lexikographisch-historische Einführung in die Sachgruppenlexikographie (S. 9-91) von H. E. WIEGAND bereichert, die das Werk in die onomasio-

1 Es wird nicht erwähnt, auf welcher Basis die prozentuale Zeitersparnis errechnet wurde. 
logische Wörterbuchlandschaft einordnet und Leser(innen) durch die Geschichte dieses Nachschlagewerkes führt. Interessierte finden zudem eine ausgewählte Bibliographie zur Lexikografie und Onomasiologie (S. 101-192), ebenfalls von H. E. WIEGAND, vor. In der methodologischen Einführung (S. 193-206), die für die Neuerarbeitung besonders wichtig ist, wird u. a. darauf eingegangen, wie das Textkorpus zusammengesetzt ist, welche Kriterien zur Auf- und Herausnahme von Wörtern und Mehrwortausdrücken ausschlaggebend sowie welche Arbeitsschritte für die Neubearbeitung nötig waren. Mit dem neu gestalteten und jetzt vollständigen alphabetischen Zugriffsregister kommt man zudem einer langjährigen Forderung der Benutzer(innen) nach. ${ }^{2}$ Auf die wesentlichen Neuerungen des Nachschlagewerkes, insbesondere die Korpusgrundlage, die inhaltlichen Erweiterungen und Aktualisierungen des Wörterverzeichnisses nach Sachgruppen sowie formale Änderungen, wird im Folgenden einzeln eingegangen. Anschließend wird in Abschnitt 5 die erstmals erschienene elektronische Version genauer untersucht.

\section{Korpusgrundlage}

Zum ersten Mal wurde mit einem zugrunde liegenden Textkorpus ${ }^{3}$ eine umfangreiche Datengrundlage geschaffen, aus der mithilfe eines computergestützten Recherche- und Analysetools mehr als 30\% neue Wörter in zum Teil neue Sachgruppen Eingang fanden und existierende Wörter hinsichtlich ihrer Aktualisierung überprüft wurden. Ein umfangreiches Textkorpus ist vor allem eine empirische Basis zur Datengewinnung, in der das Typische und Usuelle einer Sprache aufgedeckt werden kann. Für den neuen DoRNSEIFF hat dies zur Folge, dass das kompetenzgesteuerte Sammeln von sachbezogenen Wörtern seitens der Lexikograf(inn)en und somit individuelle intuitive Wortassoziationen keine Rolle mehr spielen. Sprachliche Befunde sind nicht mehr willkürlich und finden nicht mehr ,zufällig“ Eingang ins Wörterbuch, sondern sind statistisch abgesichert. Daraus ergibt sich, dass die Artikelinhalte der semantisch-ontologischen Gruppierungen, wie sie in der 8. Auflage dargestellt sind, nachweisbar und deren Buchungen begründbar sind.

Für Interessierte bietet besonders die Darstellung der korpusbasierten Methoden zur Datengewinnung und Datenauswertung (S. 193-200) einen guten Einblick in die Entstehung der neuen Auflage und in die Verfahren moderner Lexikografie im Allgemeinen. Dieser Abschnitt geht insbesondere auf die Kollokationsanalyse ein, erläutert, wie sich sinn- und sachgruppenverwandte Lexeme automatisch ermitteln lassen, und weshalb sich

2 Vgl. dazu besonders KaISER (1984: 197f).

3 Die Neubearbeitung basiert auf dem Korpus des Projekts Deutscher Wortschatz der Universität Leipzig, das zum Zeitpunkt der Auswertung rund 230 Millionen Textwörter umfasste und hauptsächlich aus Zeitungstexten zusammengesetzt ist. Wie QuAsthoff (2004: 197) bemerkt, hängt die Qualität der Ergebnisse bekanntlich vom zugrunde liegenden Korpus ab. „Im Idealfall sollte solch ein Korpus sehr groß sein, um zufällige statistische Fluktuationen auszublenden. Es sollte weiterhin möglichst ausgewogen sein, was den Anteil an verschiedenen Textsorten und Varietäten betrifft.“ Leider wird auf die genaue Korpuszusammensetzung im Buch nicht eingegangen, auch auf den angegebenen Internetseiten des Projekts erfährt man bedauerlicherweise nichts Detailliertes darüber. 
dieses Verfahren deshalb für die Erarbeitung eines Sachgruppenwörterbuchs geradezu anbietet. ${ }^{4}$ Sowohl Angaben der semantischen Nähe eines Partnerwortes zum Suchwort als auch seine Frequenz basieren auf der computergestützten Kollokationsanalyse und bieten damit bei der Neubearbeitung zum ersten Mal einheitliche Kriterien zur Auf- oder Herausnahme eines sinn- und sachgruppenverwandten Wortes, die unabhängig von subjektiven Einschätzungen der Lexikograf(innen) sind. ${ }^{5}$ Aber die methodologische Einführung lässt auch viele interessante Fragen unbeantwortet, z.B. ob das gleiche Verfahren eine Rolle für die Lemmatisierung spielt, oder welche Kriterien der Lemmatisierung zugrunde liegen. Die Frequenz spielt eine entscheidende Rolle bei der Aufnahme der Wörter in eine Sachgruppe. Ungeklärt bleibt aber, welche Frequenz ein Wort im Korpus nachweisen muss, um als Sachgruppenstichwort angesetzt zu werden. Eine gleichfalls unbeantwortete Frage ist, wie hoch der Zeitaufwand für die manuelle Nachbearbeitung war, sowohl für Bereiche, bei denen die maschinelle Unterstützung schnell Ergebnisse lieferte, wie auch in Fällen, bei denen computergestützte Analysen nicht zum gewünschten Ergebnis führten. Ferner bleibt auch die Frage offen, welche Methoden bei der Erarbeitung neuer Sachgruppen zum Einsatz kamen.

Dies wurde andererseits auch wieder schmerzhaft bewusst in Bereichen, in denen die automatischen Verfahren beispielsweise deshalb nicht funktionieren konnten, weil es entsprechende Sachgruppen bisher noch nicht oder nur rudimentär gab. Hier fehlte das Gerüst, das den automatischen Verfahren Halt bei der Einordnung gibt. (Quasthoff 2004: 196)

Dass eine Neubearbeitung nach fast 50 Jahren notwendig war, zeigen die zahlreichen Wörter und Mehrwortausdrücke, die im zugrunde liegenden Korpus bereits nicht mehr belegt sind und die viele Nutzer(innen) heute gar nicht mehr kennen dürften. Die Geläufigkeit eines Ausdrucks ist allerdings die Voraussetzung für den erfolgreichen Umgang mit diesem Nachschlagewerk. Der manuelle Aufwand zur Nachbearbeitungen der automatisch extrahierten Daten war nicht unerheblich, insbesondere bei der Einordnung der ermittelten Lexeme in passende semantische Gruppen eines Wörterbuchartikels. Fachgutachter(innen) ausgewählter Bereiche und Disziplinen erhielten automatisch extrahierte Ergebnisse, um diese anschließend genauer zu untersuchen und zu evaluieren. ${ }^{6}$ Insgesamt ist aber festzustellen, dass sich die Sachgruppen durch inhaltliche Homogenität auszeichnen, was der Datengrundlage, der computergestützten Methode und der lexikografisch-interpretatorischen Leistung der Bearbeiter(innen) zuzuschreiben ist. Nur ein digitales Korpus kann

4 Entgegen dem Vorwort (S. 7) ist es m.E. nicht verwunderlich, dass ausgerechnet einem Informatiker die Neubearbeitung des DoRNSEIFF ans Herz gelegt wurde, denn UwE QuASTHOFF ist als Informatiker bestens mit Methoden der automatischen Datenextrahierung vertraut und weiß, wie durch Kollokationsanalyse ermittelte Partnerwörter zumindest statistisch zu interpretieren sind. Den methodologischen Erläuterungen entnimmt man, dass vor allem QUASTHOFF selbst ebenfalls die lexikografischen Interpretationen durchgeführt hat, außer für einige spezielle Bereiche, für die externe Gutachter(innen) hinzugezogen wurden.

5 KAISER (1984: 192) schlägt in seiner Rezension der 7. Auflage vor, für eine Neuauflage zu prüfen „,inwieweit die Corpora des Instituts für deutsche Sprache in Mannheim, z.B. für Kontrollgänge, zu nutzen sind“. Die vorliegende 8. Auflage zeigt, dass ein Korpus heute vor allem als primäre Datengrundlage gewinnbringend genutzt werden kann und nicht nur als sekundäres Prüfinstrument im Kontrollgang herangezogen werden sollte, wie es KAISER vorschlug.

6 Für die Bereiche Literatur, Medizin, Musik, Politik, Sport und Technik wurden externe Gutachter(innen) (vor allem Linguist(innen)) hinzugezogen. 
heutzutage die einzig angemessene Datenbasis für eine umfassende Revision dieses Werkes sein, und nur ein korpusgestütztes Verfahren konnte die Neubearbeitung wesentlich schneller vorantreiben.

\section{Erweiterungen und Aktualisierungen}

Inhaltliche Erweiterungen betreffen sowohl Hauptgruppen als auch Sachgruppen. Vergleicht man die Hauptgruppen der 7. mit denen der 8. Auflage, so lassen sich grob die folgenden Modifizierungen verzeichnen:

- Umbenennungen (z.B. Hauptgruppe 1 ,Anorganische Welt. Stoffe‘ heißt nun ,Natur und Umwelt'; Hauptgruppe 2 ,Pflanze. Tier. Mensch' heißt nun ,Leben')

- Auflösung einiger Hauptgruppen, wobei deren Inhalte in anderen Hauptgruppen aufgingen (z.B. 10 ,Sinnesempfindungen“ $\rightarrow$ in 7 ,Sichtbarkeit, Licht, Farbe, Schall, Temperatur, Gewicht, Aggregatzustände' und in 16 ,Essen und Trinken')

- Inhaltliche Erweiterungen (z.B. 18 ,Wirtschaft" $\rightarrow 20$,Wirtschaft, Finanzen')

- Verschiebung einiger Sachgruppen in andere Hauptgruppen (z.B. ,Geruch und Geschmack' aus Gruppe $7 \rightarrow$ jetzt in 16 ,Essen und Trinken')

- Hinzufügen neuer Hauptgruppen (z.B. 16 ,Essen und Trinken ‘ und 17 ,Sport und Freizeit‘)

Wesentliche Erweiterungen und Neuerungen betreffen vorwiegend die Hauptbereiche Politik, Wirtschaft, Technik, Recht, Wissenschaft, Medizin und Sport und hier insbesondere die Themen Börse, Computer, Medien, Medizin, Naturwissenschaften, Sport und Verkehr. ${ }^{7}$ Einen beispielhaften Einblick in das Ausmaß der Aktualisierungen und Erweiterungen bieten die Sachgruppen der Hauptgruppe ,Geräte und Technik', die in folgender Übersicht gegenübergestellt sind. ${ }^{8}$

7. Aufl. Wohnung, Haus; Gebäudeteile; Liege- und Sitzmöbel; Kastenmöbel; Stützgeräte; Behälter für Flüssiges usw.; Behälter für Festes; Webstoffe; Bekleidung; Schmuck, Verzierungen; Hieb- und Stichwaffe; Schusswaffe; Geschoss; Abwehr und Schutz; Werkzeuge; Maschine; Elektrische Anlagen

8. Aufl. Wohnung, Haus; Gebäudeteile; Möbel; Stützgeräte; Behälter für Flüssiges und Gase; Behälter für Festes; Kleidung; Webstoffe; Schmuck, Verzierungen; Technik; Werkzeug; Maschine; Elektrische Anlagen; Elektronik; Telefon; Computer; Daten und Software; Internet; Energieversorgung; Atomenergie; Fahrzeugtechnik; Rohstoffe, Werkstoffe; Hieb- und Stichwaffe; Schusswaffe; Geschoss, Bombe; Abwehr und Schutz; Militärtechnik, Rüstung

Die Inhalte der Sachgruppen selbst, also die einzelnen Wörterbuchartikel, wurden ebenfalls aktualisiert und erweitert. Lobenswert ist, dass vor allem die vielen Regionalismen herausgenommen wurden (z.B. die Bezeichnungen für Polizei- und/oder Vollzugsbeamte: Krause (Berlin), Protscher (rhein.), Schmutzlappen (bayr.), Udel (Hamburg)), die bislang ohne

7 Insgesamt liegen in der 8. Auflage 970 Sachgruppen mit rund 90000 Wörtern und Wortgruppen vor.

8 Neu hinzugefügte Sachgruppen der 8. Auflage sind fett gedruckt. 
erkennbares System Aufnahme in den einzelnen Artikeln fanden. ${ }^{9}$ Ebenso fielen aufgrund geringer oder keiner Korpusbelegung veraltete Wörter und Mehrwortausdrücke (z.B. der aus Sachgruppe ,Kleidung': Schnürleib, Gehpelz, Beinkleid) heraus. ${ }^{10}$ Inhaltliche Auffüllung erfuhren die Sachgruppen mit aktuellen Korpusbefunden (wie etwa mit T-Shirt, Sweatshirt, Jeansjacke, Slip). ${ }^{11}$ Gesellschaftlich relevante Neuerungen waren problemlos durch ein aktuelles Korpus aufzuspüren und führten zur Lexemexpansion (mehr als 30\% neue Wörter und Wortgruppen). Die Aktualität der Einträge spiegelt sich u.a. in 21.19 ,Gesetz' durch die Aufnahme von Abtreibungsgesetz, Hochschulrahmengesetz, Lohnfortzahlungsgesetz, Rundfunkgesetz wider. Die inhaltliche Revision ist aufgrund der aktuellen und umfangreichen Korpusbasis insgesamt als sehr positiv zu bewerten, auch deshalb, weil die meisten Haupt- und Sachgruppen allgemeine Themenbereiche der öffentlichen Sprache sind und nicht fachspezifischen Diskursen entstammen. Deshalb eignet sich ein Zeitungskorpus durchaus als Wörterbuchbasis. Die Aktualisierung des DoRNSEIFF steigert gleichzeitig den Wert der vorherigen Auflage, da durch die Dokumentation dieses Sprachzustands auch der kulturelle Entwicklungsstand stärker hervortritt.

\subsection{Mikrostrukturelle Veränderungen}

Die prinzipielle Einteilung der Wörterbuchartikel in Form- und Verweisteil wurde beibehalten, dennoch gibt es wesentliche inhaltliche und formale Änderungen vor allem im Formteil. Während in der 7. Auflage Wortbildungselemente mit in den Artikel eingebaut waren, sind diese nun separat dem Formteil vorangestellt und zusätzlich mit konkreten Beispielen veranschaulicht. ${ }^{12}$ Im Formteil finden Benutzer(innen) die einzelnen Wörter innerhalb einer Begriffsreihe nach wie vor alphabetisch angeordnet, aber jede Sachgruppe wird strikt nach einem neuen asemantischen Prinzip organisiert: Am Beginn steht eine Auflistung substantivischer Gruppen, gefolgt von Adjektiven/Adverbien und verbalen Gruppierungen. ${ }^{13}$ Innerhalb dieser Ordnung stehen Einwortlexeme vor Mehrwortausdrü-

9 Wie bereits KAISER (1984) zu Recht kritisierte, handelte es sich bei den Regionalismen häufig auch um Zufallsfunde.

10 Lexeme mit einer absoluten Häufigkeit unter 10 Belegtreffern und Wortgruppen, die überhaupt nicht im Korpus belegt waren, wurden herausgenommen.

11 Ähnlich verhält es sich mit veralteten Verwandtschaftsbezeichnungen wie Muhme, Eidam, Bruderstochter und die Neuaufnahme von Junior, Pflegeeltern, Daddy, Alleinerziehende. Potenzielle Neukandidaten mussten eine Häufigkeit von mindestens 20 Belegen nachweisen.

12 In der 7. Auflage hat man z.B. die Suffixe unter dem Artikel 11.36,Wunsch“ -dul, -hörig, -istisch, -phil 9 -ismus, -lagnie, -manie, -philie, -sucht, -wut innerhalb des Artikels ohne konkrete Beispiele aufgereiht. In der 8. Auflage sieht die Wortbildungsangabe wie folgt aus:

\subsection{Wunsch}

-gier: Machtgier $\cdot$ Neugier $\cdot$ Raffgier

-manie: Egomanie $\cdot$ Gigantomanie $\cdot$ Kleptomanie

-phil: bibliophil · frankophil · pädophil

-sucht: Geltungssucht · Rachsucht · Sehnsucht

-wut: Arbeitswut $\cdot$ Sammelwut

13 Wie KAISER (1984: 193) betont, liegt der Haupteinwand gegen eine strikte Ordnung nach Wortarten darin, dass auf diese Weise Wörter einer semantischen Begriffsreihe auseinander gerissen werden. Da m.E. Benutzer(innen) einen bestimmten Ausdruck suchen, von dessen Inhalt sie be- 
cken. So genannte Kopfwörter leiten jetzt jede semantische Reihe ein, diese dienen dazu, den Suchenden schnell eine Orientierung über die inhaltlich-konzeptuelle Natur der Wortgruppe zu verleihen. Die folgende verbale Gruppe innerhalb der Sachgruppe ,Empfindung‘ soll als Illustrierung dienen:

7. Auflage 11.4: ahnen, apperzipieren, bemerken, empfinden, erleben, (sich) fühlen, merken, reagieren, spüren, verspüren.

8. Auflage 10.4: empfinden, ahnen, bemerken, erleben, fühlen, merken, reagieren, spüren, verspüren.

Dass die Kopfwörter eine Signalwirkung haben und aus der üblichen alphabetischen Ordnung herausragen, dürfte aufgrund mangelnder typografischer Hervorhebung der Aufmerksamkeit einiger Nutzer(innen) entgehen. Ihre Funktion und ihre gesonderte Stellung sollten aber sofort erkennbar sein, und man sollte diese nicht erst durch das Lesen der Benutzerhinweise bemerken. In den Benutzungshinweisen vermisst man auch Erklärungen darüber, welche Rangfolge bei den einzelnen semantischen Gruppen eines Artikels vorliegt. Beginnt die Auflistung mit hyperonymen Partnerwörtern oder handelt es sich bei der ersten Gruppe immer um die statistisch häufigsten Kontextpartner? Gerade bei der Korpusbasiertheit und bei dem Einsatz statistischer Verfahren stellt sich die Frage, ob die Rangfolge der einzelnen semantischen Gruppen innerhalb einer Wortart bestimmten Kriterien unterliegt. ${ }^{14}$

Der Verweisteil erfuhr in erster Linie eine inhaltliche Überarbeitung. Jeder dort aufgeführte Artikel steht in einem engen inhaltlichen Zusammenhang mit dem Ausgangsartikel. ${ }^{15}$ Diese Verweise sind besonders dann nützlich, wenn Nutzer(innen) einen bestimmten Ausdruck im Ausgangsartikel nicht finden konnten und sie deshalb weitere Artikel mit semantisch eng verwandtem Bezug konsultieren möchten. Dabei sind die Voraussetzungen für einen Verweis unterschiedlich. Es liegen u. a. paradigmatische, syntagmatische, assoziative, logisch-kausale oder thematisch-diskursive Relationen zwischen den vernetzten Wörtern vor. ${ }^{16}$ Für das Anlegen eines Verweises liegen Kriterien zugrunde, die in der methodologischen Einführung wie folgt erläutert werden:

Sind zwei Wörterbuchartikel durch solch eine semantische Verwandtschaft verbunden, so enthalten sie in der Regel mehrere Wörter oder Wortgruppen gemeinsam. Damit bot sich eine Möglichkeit, mit automatischen Mitteln Vorschläge für neue Verweisangaben zu erzeugen: Enthalten nämlich zwei Wörterbuchartikel mehrere Wörter oder Wortgruppen gemeinsam, sind möglicherweise Verweise zwischen diesen Artikeln sinnvoll. Als angebracht erwies sich eine Minimalzahl

reits eine Vorstellung haben und somit über eine Vorstellung der gewünschten Wortart verfügen, bietet sich eine Anordnung nach Wortarten, wie sie in der 8. Auflage angelegt wurde, an.

14 Insbesondere bei substantivischen und adjektivischen Gruppen ist keinerlei Kriterium erkennbar. Als Ausnahme dazu: „Über die semantischen Gruppen hinweg findet man dagegen oft wortfamilienintern assoziierte Ausdrücke. Wo dies sinnvoll erschien, wurden diese auch als erste Einträge in den semantischen Gruppen gewählt, wie bei 2.31 Erholung: Erholung $[\ldots] \cdot$ erholt $[\ldots] \cdot$ erholsam [...] · erholen [...].“(QUASTHOFF 2004: 206).

15 Während einige Verweise in der 7. Auflage z.T. unergiebig waren (vgl. KAISER 1984: 195), ist der semantische Bezug zwischen den ausgewiesenen Artikeln nachvollziehbar. Folgt man den Verweisen, wird i.d.R. die vermutete Sachgruppenverwandtschaft bestätigt.

16 Die unterschiedlichen Beziehungen werden anhand folgender Verweise deutlich: z.B. Relation der Bedeutungsgleichheit (Verbot $\leftrightarrow$ Nichtberechtigung), Relation des Bedeutungsgegensatzes (süß↔ sauer), Relation der Über- und Unterordnung (Fußball $\leftrightarrow$ Sport), typische Eigenschaften (Gebäudeteile $\leftrightarrow$ hoch) etc. 
von vier gemeinsamen Wörtern oder Wortgruppen, um einen Verweisvorschlag zu erzeugen. (QUASTHOFF 2004: 201)

Eine Frage bleibt hier unbeantwortet: Handelt es sich laut des obigen Zitats beim Verweissystem immer um ein bidirektionales Verweisen oder ist gegenseitiges Verweisen nur eine Option, und es war durchaus auch das Ziel, unidirektionale Verweise anzulegen? Wenn beim Verweisen zwischen Artikeln immer mindestens vier gemeinsame Partner vorhanden sein müssen, dann liegt trotz automatischer Erzeugung und Berechnung der gemeinsamen Wörter eine hohe Fehlerquote vor. So verweisen die Artikel 2.13 ,Mann“ und 2.14 ,Frau“ sowie 16.13 ,süß` und 16.14 ,sauer` gegenseitig aufeinander, obwohl sie über keinerlei gemeinsame Wörter verfügen. Nur einen gemeinsamen Partner (höllisch) besitzen die Artikel 22.8 ,Teufel` und 22.10 ,Unterwelt, Hölle‘. War es tatsächlich die Intention, auch unidirektionale Verweise einzuarbeiten (etwa ein Fünftel aller Verweise ist tatsächlich unidirektional), dann bleiben die Kriterien zum Anlegen dieser Verweise unklar. ${ }^{17}$

\subsection{Vollständiges alphabetisches Register}

Neben einem alphabetischen Sachgruppenregister (S. 226-231) wurde der neue DoRNSEIFF nun auch mit einem vollständigen alphabetischen Zugriffsregister (S. 463-933) ausgestattet, das sowohl Sachgruppenwörter als auch alle Artikelinhaltswörter beinhaltet. Schlägt man ein Wort dort nach, erfährt man nicht nur dessen zugehörige Sachgruppennummer, sondern auch den entsprechenden Sachgruppentitel. Dadurch lässt es sich im Gegensatz zum Zugriffsregister der 7. Auflage (welches unvollständig und praxisfern war, sich auf die Angabe der Sachgruppennummer beschränkte und auf zugehörige Sachgruppentitel verzichtete) in der Tat als ein orientierendes Hilfsmittel bezeichnen. ${ }^{18}$ Vor allem bei Wörtern, die zahlreichen Sachgruppen zugeordnet sind, ermöglicht es einen gezielten Zugriff, da die gewünschte Sachgruppe eines gesuchten Wortes schneller identifizierbar ist. Der folgende Ausschnitt demonstriert die Registerangaben für das Wort Entwicklung:

4.3 ,Wachsen‘; 5.24 ,Veränderung‘; 5.26 ,Allmähliche Entwicklung‘; 5.39 ,Erzeugung;; 18.27 ,Kulturelle Entwicklung'; 18.29 ,Krieg‘.

\section{Layout}

Der aktuelle DoRNSEIFF besticht mit seinem neuen, schlichten und äußerst benutzerfreundlichen Layout. Bereits auf der Umschlagseite können sich Benutzer(innen) sehr anschaulich über den Umgang mit dem Wörterbuch und seinen unterschiedlichen Zugriffsstrukturen,

17 Da keine Kriterien für einseitige Verweise dargelegt werden, bleibt die Frage, warum z.B. Artikel 20.47 ,Autoindustrie“ auf Artikel 20.46 ,Industrie' verweist, aber Artikel 20.48 ,Pharmaindustrie“ nicht. Bei vielen einseitigen Verweisen liegen keine gemeinsamen Partnerwörter vor, z.B. 16.19 ,Geruch' $\rightarrow 16.12$,Geschmack', 7.28 ,Geräusch' $\rightarrow 14.11$,Musik', 16.1 ,Hunger, Durst' $\rightarrow 20.6$ ,Armut', 3.42 ,stumpf $\rightarrow 9.58$, minderwertig'.

18 Vgl. KaISERs (1984: 197f.) Kritik am alphabetischen Zugriffsregister der 7. Auflage. 
über die ein Ausdruck auffindbar ist, informieren. Der besseren Übersichtlichkeit dient die klare optische Trennung zur Zweiteilung der Wörterbuchartikel in Verweis- und Formteil. Eine dem Formteil vorangestellte Information zur Wortbildung ist leicht durch die deutliche Abtrennung erkennbar. Eine benutzerfreundliche Orientierungshilfe bietet auch die neu gestaltete Kopfleiste, insbesondere innerhalb des eigentlichen Wörterbuchteils, in der auf der linken Buchseite die aufgeschlagene Hauptgruppennummer und der Hauptgruppentitel stehen, während auf der rechten Buchseite Sachgruppennummer und -titel der dort befindlichen Sachgruppen festgehalten sind. Hier wurde an eine zusätzliche Benutzerführung gedacht. Nachdem man über das Zugriffsregister die entsprechende Nummer der relevanten Sachgruppe herausgesucht hat, kann man sich jetzt beim Nachschlagen an der Kopfleiste besser orientieren. Die Kopfleiste bietet zudem eine Orientierung beim Folgen von Artikelverweisen.

\section{CD-ROM-Version}

Mit dem Erscheinen der 8. Auflage liegt der DoRNSEIFF auch erstmals als elektronische Version auf CD-ROM vor. Es handelt sich dabei um eine rein maschinelle Umsetzung des Druckwörterbuchs, die formal und inhaltlich mit diesem fast identisch ist. ${ }^{19}$ Abbildung 1 bietet einen ersten Einblick in das CD-ROM-Nachschlagewerk.

Vertraute Nutzer der Druckversion werden die Inhalte der einzelnen Rahmen schnell einordnen können. Der linke Rahmen ist das Inhaltsfenster, welches das Inhaltverzeichnis des Buches darstellt, und durch welches Benutzer(innen) mühelos durch das „Buch“ navigieren können. Im Hauptrahmen, dem so genannten Dokumentfenster, erkennt man die entsprechend ausgewählte Stelle des Buches, hier Sachgruppe 18.25 ,Freiheit ${ }^{`}$ innerhalb der Hauptgruppe 18 ,Gesellschaft'. Das schmale Fenster oberhalb des Dokumentfensters zeigt den Pfad des Inhaltsverzeichnisses an, und das unterste Fenster, das sich horizontal über den gesamten Bildschirm erstreckt, dient der Aufreihung der Suchergebnisse. ${ }^{20} \mathrm{Be}-$ steht der Wunsch, Fenster auszublenden, um sich auf bestimmte Informationen zu konzentrieren, z.B. den Wörterbuchartikel so groß wie möglich darzustellen oder nur das Inhaltsverzeichnis als aufklappbare Struktur anzuzeigen, so kann die Ansicht gewechselt werden (siehe die sechs Reiter am linken unteren Rand). In der obigen Abbildung dagegen wurde die Ansicht „Alle“ ausgewählt - eine komfortable Ansichtsoption, in der alle relevanten Informationen gleichzeitig miteinander verknüpft erscheinen, während in den anderen Ansichten eine individuelle Informationsfokussierung möglich ist, da nur bestimmte Inhalte sichtbar werden.

19 Auf das alphabetische Zugriffsregister wird verzichtet, da bei einer elektronischen Version dafür i.d.R. kein Bedarf besteht.

20 Das Suchwort im Illustrationsbeispiel (Abb. 1) ist frei. Die Fundstellen sind im Dokument selbst gekennzeichnet und im entsprechenden Trefferfenster mit ihren zugehörigen Fundstellen nach Haupt- und Sachgruppe geordnet aufgelistet. 


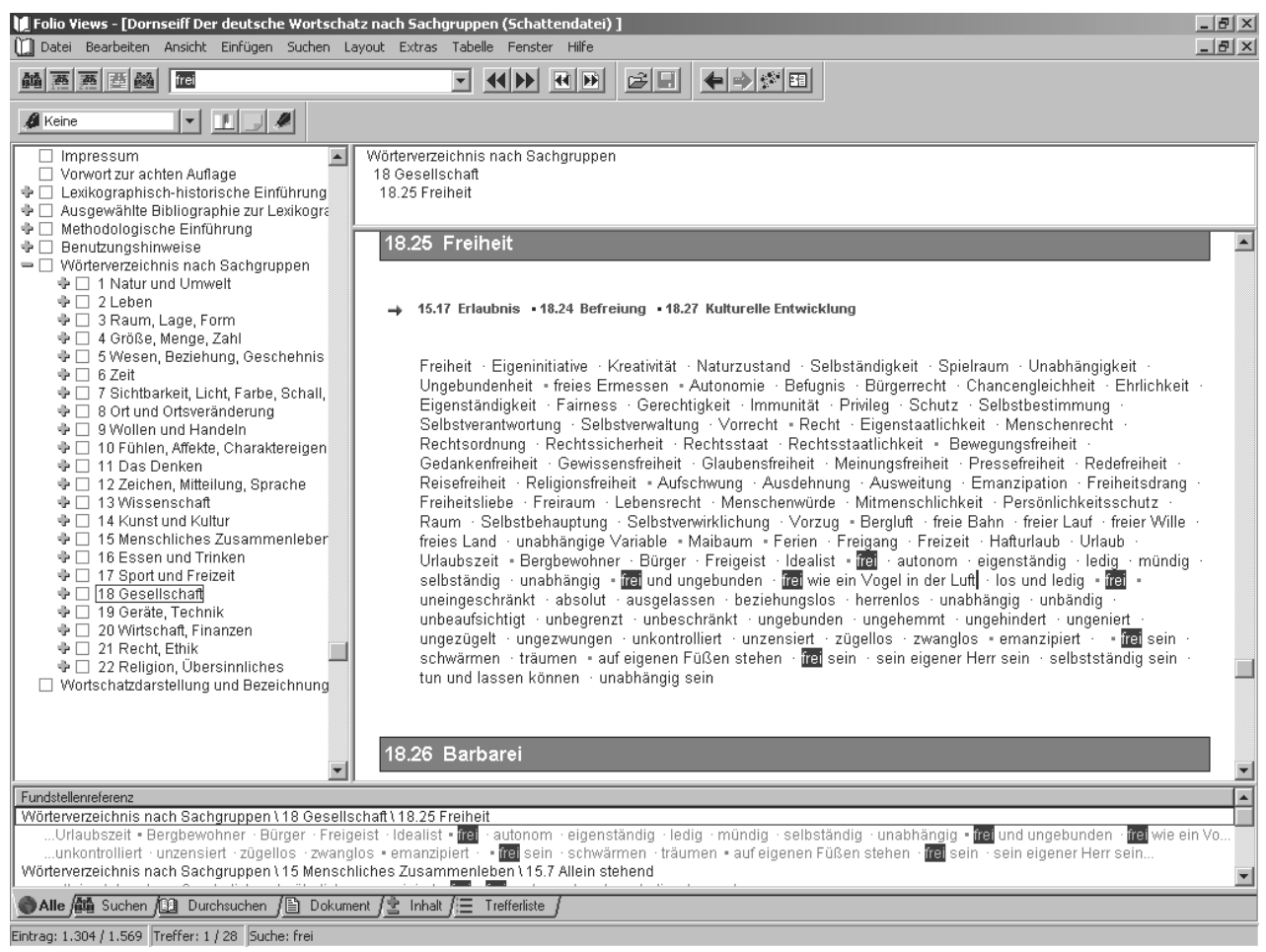

Abb. 1: Ansicht des Artikels 18.26 Freiheit im elektronischen DorNSEIFF

\subsection{Daten-Retrieval}

Der größte Vorteil des elektronischen DoRNSEIFF liegt in der Möglichkeit, schnell und gezielt Datenabfragen durchführen zu können. Dafür sorgt die Integration der Software FolioViews, eine Software zur Informationsabfrage, die eigens für eine schnelle, gezielte und effektive Suche nach Wörtern in umfangreichen und strukturierten Dokument- oder Textsammlungen entwickelt wurde. Während geübte Nutzer(innen) von elektronischen Nachschlagewerken schnell die Möglichkeiten allgemeiner Suchanfragen erfasst haben, muss man sich für detaillierte Auskünfte im Hilfemenü, in dem umfangreiche Benutzungshinweise vorzufinden sind, orientieren. Obwohl einige Softwarekomponenten allgemein bekannt sind, ist es notwendig, sich mit der speziellen FolioViews-Terminologie vertraut zu machen. Das größte Manko der im Hilfemenü befindlichen Benutzerhinweise besteht aber darin, dass diese für das Programm FolioViews geschrieben sind, und nicht mit speziellen Gebrauchshinweisen für den elektronischen DoRNSEIFF abgestimmt wurden. ${ }^{21}$ Einfache

21 Die Software ist eine reine Daten-Management-Lösung, die ursprünglich zur Informationsorganisation und zum Information-Retrieval für Wirtschaftsunternehmen entwickelt wurde. Sie ist entsprechend allgemein oder mitunter umständlich formuliert oder enthält für den DoRNSEIFF irrelevante Funktionsbeschreibungen. 
Benutzerfragen des Typs Wie sucht man ein Wort im gesamten Wörterverzeichnis? oder Wie beschränkt man seine Suche auf eine Sachgruppe? sind nicht zu finden. Kommt man damit nicht zurecht, steht einem nur die Kurzerläuterung auf der Innenseite der CD-Hülle zur Verfügung. Diese ist als Einstieg empfehlenswert, da die Beschreibung in aller Kürze über die wichtigsten Funktionen informiert. Ein benutzerfreundliches Hilfeprogramm mit integrierter Bedienungserklärung, die anhand konkreter sprachlicher Beispiele den Umgang mit dem elektronischen DoRNSEIFF erläutert, bleibt für die nächste Auflage zu wünschen.

Für alle, die lediglich schnell nach sinnverwandten Wörtern eines Ausdrucks suchen, also die Artikel einer bestimmten Sachgruppe schnell aufrufen wollen, bedarf es keiner tiefen Sachkenntnisse der Software. Die Suchabfrage kann über das sofort sichtbare Eingabefenster „Suche“ der Benutzeroberfläche oder über die intuitiv erfassbare Menüoption „Suche“ erfolgen. Darüber hinaus lässt sich eine Suche über entsprechende Buttons der Symbolleiste durchführen. Die besondere Stärke der elektronischen Version gegenüber dem Printprodukt liegt vor allem in den unterschiedlichen Suchoptionen (,Suche“, „Erweiterte Suche“, „Detailsuche“, „Einschränkende Suche“), ${ }^{22}$ deren vorgefertigte Suchmasken die groben Unterschiede zwischen den einzelnen Suchoptionen recht schnell erkennbar machen. Für das vollständige Erfassen aller Suchmodalitäten müssen Ungeübte etwas Einarbeitungszeit aufwenden. So können Nutzer(innen) beispielsweise gezielt nach einem Lexem oder einem Mehrwortausdruck suchen und dabei die Suche auf einen bestimmten Buchausschnitt, Hauptgruppenbereich oder auf eine Sachgruppe beschränken. Darüber hinaus ist es ihnen möglich, durch die Anwendung der Booleschen Operatoren eine Suchverknüpfung von mehreren Wörtern vorzunehmen, oder z.B. durch Platzhalterzeichen nach Komposita mit einem bestimmten Grund- oder Bestimmungswort zu suchen. Außerdem lässt sich die Suche auf verschiedene Wortarten oder Personenbezeichnungen einschränken. Die Suche ist nicht auf das Wörterverzeichnis nach Sachgruppen beschränkt, so dass interessierte Nutzer(innen) auch in allen im Buch befindlichen Erläuterungstexten recherchieren können. Da das Tool über ein integriertes Textverarbeitungsprogramm verfügt (siehe dazu Abschnitt 5.3), sind auch Spezialrecherchen innerhalb der eigens angelegten Markierungen und Anmerkungen möglich. Die Präsentation der Suchergebnisse erfolgt mit bemerkenswerter Schnelligkeit, was auch daran liegt, dass die CD-ROM-Version beim Installieren eine Kopie des DoRNSEIFF auf der Festplatte anlegt.

Leider verfügt die elektronische Version nicht über eine schreibungstolerante Suche, so dass bei Falschschreibung die Suche erfolglos bleibt. Ebenso erfolglos können Suchanfragen sein, wenn diese eine orthografisch zulässige Schreibvariante enthalten, die aber nicht im DoRNSEIFF gebucht ist. ${ }^{23}$ Da die Unterscheidung zwischen Klein- und Großschreibung prinzipiell nicht erfolgt, muss die Suche in entsprechenden Fällen über die Eingrenzung der Wortart definiert werden. Auch nach flektierten Formen kann im Wörterverzeichnis nach Sachgruppen nur über die Kopplung mit Platzhalterzeichen für Wortformen gesucht werden, das direkte Eingeben der flektierten Form in der Suchmaske erbringt dort i.d.R. keine

22 Suchoptionen, die über die einfache Suche hinausgehen, sind sowohl über das Menü als auch über die Symbolleiste aufrufbar. Da es sich um identische Suchbefehle handelt, ist es erstaunlich, dass die im Menü befindliche Funktion „Einschränkende Suche“ in der Symbolleiste als „Suchmaske 2 " bezeichnet ist, alle anderen Bezeichnungen sind identisch.

23 Sucht man nach den orthografisch zulässigen Formen Jogurt, Club, Greuel werden keine Treffer erzielt, Joghurt, Klub, Gräuel sind dagegen im DoRnSEIFF gebucht. Mit einer fehlertoleranten Suche ließe sich dieses Problem vielleicht beheben. 
Treffer. ${ }^{24}$ Verläuft die Suche erfolgreich, ist die Darstellung der Suchergebnisse vorbildlich. Zum einem sind die Treffer deutlich im Wörterbuchartikel markiert (siehe Abbildung 1), zum anderen gibt es mehrere Möglichkeiten, sich die Treffer übersichtlich anzeigen zu lassen, z.B. geordnet nach Haupt- und Sachgruppe. In einer kompletten Trefferliste lässt sich komfortabel durch direktes Anklicken eines Treffers über Menübefehle oder durch Betätigung der Symbolfunktionen (Pfeil-Buttons) zu den nächsten oder vorherigen Treffern navigieren. Einmal gestellte Suchanfragen lassen sich über den Historienüberblick erneut einsehen, die Ergebnisse früherer Suchanfragen sind immer wieder abrufbar.

\subsection{Verlinkung}

Einen weiteren großen Vorteil des elektronischen Mediums bieten die Verlinkungsmöglichkeiten, welche aber nur zum Teil ausgeschöpft wurden. So spricht QUASTHOFF in der methodologischen Einführung davon, dass mit einer elektronischen Version eine angemessene Orientierungsmöglichkeit bereitstehe, bei der die Vernetzung zwischen Sachgruppen sinnvoll und nachvollziehbar ist:

Die Umsetzung der komplexen semantischen Zusammenhänge zwischen den Bezeichnungen der Sachgruppen in eine lineare Abfolge ist immer problematisch und wird auch die subjektive Sicht des Autors wiedergeben. Im Zeitalter von Internet und Hypertext verliert allerdings die im gedruckten Buch notwendige lineare Abfolge ihre zentrale Bedeutung und wird ersetzt durch die Forderung nach einer sinnvollen und nachvollziehbaren Vernetzung der einzelnen Sachgruppen. In diesem Sinne schafft die elektronische Version einer solchen Sammlung erstmals die angemessenen Orientierungsmöglichkeiten. (QUASTHOFF 2004: 202)

Da die elektronische Version völlig am gedruckten Vorbild ausgerichtet ist, bietet sie keine dem Medium angemessene Orientierungsmöglichkeit, die über die Druckversion hinausgeht. Die im Print-DoRNSEIFF vernetzten Strukturen liegen in Form von Artikelverweisen vor. Während es aufwändiges Nachblättern erfordert, diesen Verweisen zu folgen, kann man sich im elektronischen DoRNSEIFF bequem per Mausklick direkt zur einer ausgewiesenen Sachgruppe, die typografisch hervorgehoben ist, begeben. Diese Möglichkeit allein erspart viel Zeit, die Vernetzung von Sachgruppen ist aber weder sinnvoller (denn sie ist identisch mit dem Buch) noch deutlicher (denn es liegt außer einer farblichen Hervorhebung keine andere Gestaltung zugrunde). Der weitere Ausbau von Verknüpfungsmöglichkeiten könnte z.B. darin bestehen, dass Wörter eines Artikels, die selbst als Stichwort gebucht sind, als Link realisiert werden. ${ }^{25}$

\subsection{Textverarbeitungselemente}

Ein dritter großer Vorteil des CD-Nachschlagewerkes ist das integrierte Textverarbeitungssystem von FolioViews, mit dem Benutzer(innen) spezielle Information nach individuellen

24 Die Suche einer flektierten Form ist dagegen im Textbereich des ,Buches' möglich, nur im eigentlichen Nachschlagewerk muss dafür ein spezieller Operator eingesetzt werden.

25 Das Wort töten in den Artikeln 5.37 ,Schwäche' und 5.42 ,Zerstörung' könnte als Link zum Artikel 2.41 ,Töten' gesetzt werden. 
Bedürfnissen gestalten können. Voraussetzung für die aktive Gestaltung ist das Anlegen einer „Schattendatei“ von einer so genannten Infobase (Originaldatei). In dieser können dann z.B. ganze Wörterbuchartikel gekennzeichnet, Lesezeichen angelegt, bestimmte Wörter unterschiedlich markiert und Anmerkungen mithilfe von Notizzetteln vorgenommen werden. Der Vorteil dabei ist, dass diese Schattendateien individuelle Markierungen enthalten, das Wörterbuch selbst aber unverändert bleibt. Ist man mit dem Anlegen von Schattendateien vertraut, bereitet der Umgang mit dem Gestaltungstool in seinen groben Zügen kaum Probleme, da die meisten Funktionen von üblichen Textverarbeitungsprogrammen her bekannt sind. Das elektronische Nachschlagewerk kann somit als individuelles Arbeitswerkzeug funktionieren. Textmarker, ${ }^{26}$ Lesezeichen, Grafiken und Notizzettel lassen sich mühelos in gefundene Artikelstellen einbauen, um bestimmte Informationen zu gruppieren oder einfach nur um diese zu einem späteren Zeitpunkt mühelos wieder zu finden. Abbildung 2 demonstriert einige der Gestaltungsmöglichkeiten. ${ }^{27}$

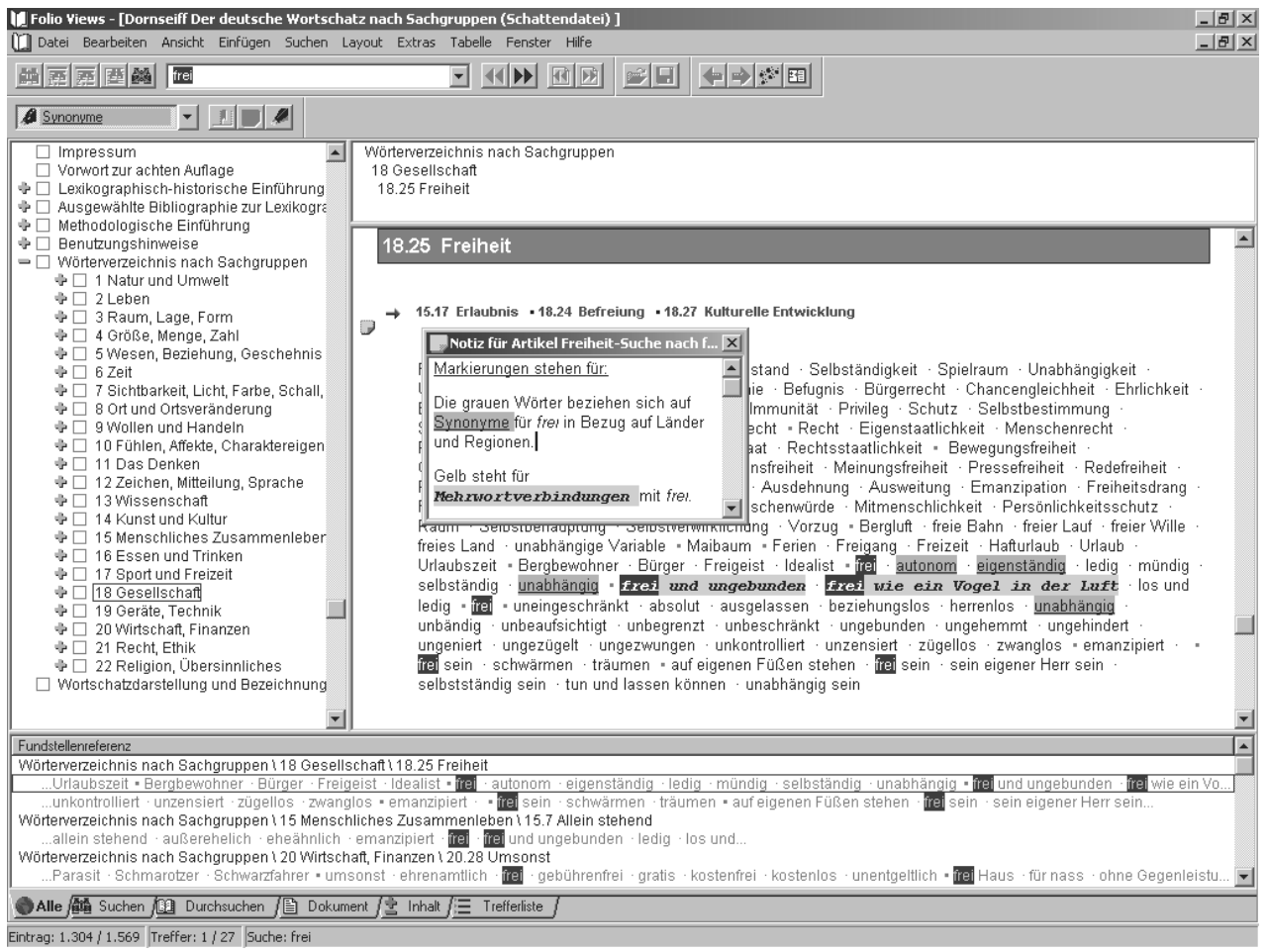

Abb. 2: Textverarbeitungselemente

26 Die Definition von Textmarkierungen erfolgt z.B. durch Wahl der Vorder- und Hintergrundfarben, Schriftarten und -größe, Unterstreichungen, Hervorhebung, Kursivierung etc.

27 So wurde z.B. das Wort frei gesucht. Markiert wurden hier Mehrwortausdrücke und Synonyme, die sich im Zusammenhang mit Ländern oder Regionen verwenden lassen. Ein Notizzettel dokumentiert die Markierungsüberlegungen. 
Die Möglichkeiten, Markierungen und Anmerkungen individuell zu benennen, ${ }^{28}$ erleichtert auch die von der Software gegebene Option, innerhalb der eigenen Kommentare und Markierungen gezielt zu suchen, was als besonders benutzerfreundlich zu bewerten ist. Aber auch innerhalb der Textverarbeitungsmöglichkeiten gilt, dass es zur Aneignung tieferer Sachkenntnis im Umgang mit der Software und um die einzelnen Programmkapazitäten ausschöpfen zu können, eines äußerst gründlichen Einlesens in die sehr umfangreichen und z.T. zu allgemein gehaltenen Benutzerhinweise bedarf.

Zusammenfassend lassen sich in der elektronischen Version im Wesentlichen folgende Vorteile erkennen: die schnellen und vielfältigen Retrievalmöglichkeiten, die Wahl der Informationsfokussierung durch unterschiedliche Ansichtsmöglichkeiten, die individuelle Gestaltung und Hervorhebung mittels eines Textverarbeitungstools, die schnelleren und direkteren Wege von Verweisen und das problemlose Navigieren durch das ,Buch“. Ausbaufähig dagegen bleibt das Verlinkungssystem und die Integration einer fehlertoleranten Suche. Wünschenswert wären zusätzlich auf den DoRNSEIFF abgestimmte Benutzungshinweise, die im Hilfemenü unterzubringen wären. ${ }^{29}$

\section{Bibliographie}

\subsection{Wörterbücher}

Dornseiff $1970=$ Franz DornseifF: Der deutsche Wortschatz nach Sachgruppen. 7. Auflage. Berlin 1970.

Dornseiff 2004 = Franz DornseifF: Der Deutsche Wortschatz nach SaChgrupPen. 8., völlig neu bearbeitete und mit einem vollständigen alphabetischen Zugriffsregister versehene Auflage von Uwe Quasthoff. Mit einer lexikographisch-historischen Einführung und einer ausgewählten Bibliographie zur Lexikographie und Onomasiologie von Herbert ERNST WIEgAND. CD-ROM. Berlin/New York 2004.

\subsection{Literatur}

Hass-Zumkehr 2001 = Ulrike Hass-Zumkehr: Deutsche Wörterbücher. Berlin/New York 2001.

KaISER 1984 = STEPHAN KaISER: Der neue DornseifF: Konzept einer Neubearbeitung von Franz Dornseiffs „Der deutsche Wortschatz nach Sachgruppen“, In: Zeitschrift für Germanistische Linguistik 12, 1984, 181-199.

KLOSA 2001 = ANNETTE KLOSA: Qualitätskriterien der CD-ROM-Publikationen von Wörterbüchern. In: Ingrid Lemberg, Bernhard Schröder, Angelika Storrer (Hrsg.): Hypertext, Internet und SGML/XML für die Produktion und Publikation digitaler Wörterbücher. Tübingen 2001 ( $L e-$ xicographica. Series Maior 107), 93-101.

28 Siehe z.B. in Abbildung 2 links oben. Der grau unterlegte unterstrichene Textmarkierungstyp wurde ,Synonyme` genannt, der Notizzettel wurde mit der Überschrift ,Notizzettel für den Artikel Freiheit - Suche des Wortes frei' versehen.

29 Bezüglich allgemeiner Qualitätskriterien zur Bewertung elektronischer Nachschlagewerke siehe auch KLOSA (2001) und LeHR (1996). 
LEHR 1996 = ANDREA LEHR: Zur neuen Lexicographica-Rubrik „Electronic Dictionaries“. In: Lexicographica 12, 1996, 310-317.

QuASTHOFF 2004 = UwE QuASTHOFF: Methodologische Einführung. In: DoRnSEIFF - Der DEUTSCHE Wortschatz NaCh Sachgruppen. 8. Auflage. Berlin/New York 2004, 191-206. 